\title{
Aprender haciendo: Uso de una estrategia didáctica en un curso avanzado de la carrera de Farmacia de la Universidad de Costa Rica
}

\section{Learning with action: Using a didactic strategy in an advanced Pharmacy course at the School of Pharmacy of the University of Costa Rica}

\author{
Angie León-Salas \\ Universidad de Costa Rica \\ Facultad de Farmacia \\ San José, Costa Rica \\ angieleonsalas@gmail.com
}

Recibido: 20 noviembre 2013 Aceptado: 9 abril 2015 Corregido: 11 mayo 2015

\begin{abstract}
Resumen: El presente artículo describe la implementación de la estrategia didáctica del aprendizaje orientado en proyectos (AOP) con el objetivo de promover el aprendizaje de aspectos básicos de prevención de la enfermedad y promoción de la salud, lo anterior con el fin de impulsar el desarrollo de habilidades para la participación en intervenciones de salud pública. Estudiantes del último año de la carrera de Licenciatura en Farmacia de la Universidad de Costa Rica realizaron, durante 3 semanas, un proyecto en el cual identificaron y abordaron un problema de salud que afectaba a su grupo de clase. Se incluyó el desarrollo de una intervención por parte del estudiantado y la respuesta de cuestionarios en línea que evaluaron su propia percepción antes y después de la implementación de la estrategia. La retroalimentación brindada por el estudiantado fue positiva y apoya el desarrollo de acciones dinámicas para fortalecer el proceso de aprendizaje. Se concluye que el uso del AOP permitió analizar los contenidos teóricos de una manera aplicada. Es necesario hacer consideraciones de tiempo y evaluación con el fin de darle continuidad a la actividad propuesta.
\end{abstract}

Palabras claves: Estrategia didáctica, salud pública, farmacia.

\begin{abstract}
This study describes the implementation of Project-Based Learning (PBL) with the aim of promoting learning of basic aspects of disease prevention and health promotion to foster the development of skills to increase participation in public health interventions. During 3 weeks, senior pharmacy students at the University of Costa Rica, worked on a project that addressed a health-related problem they considered was affecting their group. Students worked on the development of an intervention and they answered an on-line questionnaire before and after the implementation of PBL. Positive feedback was provided by students and it supports the development of dynamic actions to strength the learning process. We conclude that using PBL allowed the application of actions to support theory. It is necessary to make time and evaluation arrangements in the design of this project in order to continue and improve the strategy.
\end{abstract}

Key words: Didactic Strategy, Public Health, Pharmacy

1 Farmacéutica de la Universidad de Costa Rica y Master en Salud Pública de la Universidad de Kansas. Actualmente labora en la Facultad de Farmacia de la Universidad de Costa Rica como docente invitada donde participa en los cursos: Salud Pública, Introducción a la Farmacia, Toxicología y Atención Farmacéutica. Durante su formación en Estados Unidos enfocó su labor en la participación en estudios clínicos para la cesación del uso del cigarro. Ha participado en múltiples conferencias nacionales e internacionales donde destaca un total de 10 presentaciones de poster. Su artículo Participación y opiniones de farmacéuticos costarricenses en la cesación del uso del tabaco, publicado en la Revista Costarricense de Salud Pública es pionero en la identificación de la labor del profesional de farmacia en material del consumo de tabaco. 
El curso Elementos de Salud Pública (FA-5026) se encuentra ubicado en el décimo y último ciclo lectivo de la carrera de Licenciatura en Farmacia de la Universidad de Costa Rica. Este curso se caracterizó, en el año 2012, por ser impartido por una cátedra de 3 docentes, en horario de 5 a 8 de la noche y con la asistencia a clases no obligatoria. Se debe destacar que el bloque en el cual se encuentra FA-5026 es uno de los más demandantes para el estudiantado, dada la carga académica que presentan los diversos cursos que deben matricular.

Tal y como lo detallan Escribano y Del Valle (2008), la estrategia didáctica del aprendizaje orientado en proyectos (AOP) permite al estudiantado ser partícipe y a la vez líder en el proceso de aprendizaje. Mencionan, además, que esta estrategia permite que el estudiantado tome la iniciativa en la resolución de problemas reales. Por otro lado, utilizando un enfoque constructivista, esta metodología fomenta el trabajo interdisciplinario y la búsqueda de aspectos innovadores, lo cual permite la obtención de un mayor beneficio durante el proceso de aprendizaje donde se afianzará en la construcción de ideas y conceptos, así como la interacción con diversidad de profesionales, por medio de la utilización de conocimientos actuales y previos (Rodríguez-Sandoval, Vargas-Solano, y Luna-Cortés, 2010).

Se ha indicado la necesidad de que la profesión en salud desarrolle competencias transversales interpersonales en acciones que incluyan el fomento del trabajo en equipo y el respeto hacia las acciones de sus miembros (Baños y Pérez, 2005). Este aspecto es una de las características del AOP, lo cual permite que esta metodología sea utilizada en la formación de profesionales en farmacia, principalmente en la familiarización del estudiantado con situaciones reales que deberá afrontar al finalizar su formación. Por ejemplo, Fuhrman, Buff, Eaddy y Dollar (2001) presentan el uso de tecnologías de comunicación para que estudiantes resuelvan casos de "pacientes virtuales" de manera semanal; esto, con el fin de aumentar los niveles de habilidades a nivel profesional o práctico (Fuhrman et al., 2001). De igual forma, la simulación de proyectos ha permitido que estudiantes de farmacia, en un curso de farmacia geriátrica, tomen conciencia de las dificultades que pueden enfrentar las personas adultas mayores polimedicadas en el tratamiento y adherencia a regímenes de medicación usualmente complicados (Divine y Cain, 2009).

Es importante recalcar que la vinculación de profesionales en farmacia dentro de las comunidades para favorecer el uso racional de medicamentos y promover la salud es un tema que debe ser parte integral de la formación y ejercicio profesional en farmacia (OMS, 2013). La integración del especialista en medicamentos dentro de los equipos de salud y su importancia para el tratamiento y prevención de enfermedades crónicas ha sido descrita por varios autores (Tan, Stewart, Elliott y George, 2014). De igual forma, se conoce la existencia del interés por aumentar su liderazgo y la apertura de espacios para una mayor participación en la promoción de la salud de sus pacientes (Dobson, Taylor, Henry, Keegan y Forbes, 2009).

Dada la necesidad de fortalecer habilidades que permitan una mayor integración del farmacéutico o farmacéutica dentro de acciones de promoción en salud y prevención de la enfermedad, se consideró la estrategia didáctica del AOP para abordar el tema con mayor motivación por parte del estudiantado y para la solución de una pregunta o problema real que se encuentre relacionada con el entorno en el cual se desenvolverán en el futuro (Blumenfeld et al., 1991). Este acercamiento a la realidad permite la consideración de esta estrategia metodológica 
para presentar conceptos básicos de salud pública de una forma agradable y fue, además, clave para motivar al estudiantado a presentarse a las sesiones. Lo anterior siempre vislumbrando un espacio que incitara la creatividad, la resolución de proyectos, el trabajo en equipo y el uso de conocimientos previos para la identificación y resolución de problemas específicos.

El objetivo de este estudio fue promover el aprendizaje de aspectos básicos de la prevención de la enfermedad y la promoción de la salud mediante el uso de habilidades para el desarrollo de intervenciones de salud pública con la estrategia didáctica del aprendizaje orientado en proyectos.

\section{Metodología}

Este estudio se realizó con un enfoque constructivista, se utilizó, para su desarrollo, la estrategia didáctica del aprendizaje orientado en proyectos. El proyecto tuvo una duración total de tres semanas. Durante la primera semana la docente dio a conocer las acciones a realizar así como los objetivos propuestos; se indicó que el objetivo era promover el aprendizaje de aspectos básicos de prevención de la enfermedad y promoción de la salud por medio de la realización de una intervención de salud pública en un tema real, con lo cual se esperaba tuviera un beneficio en su futuro desempeño como profesionales en salud.

Se inició con la organización del estudiantado en grupos de cinco a siete personas. Se solicitó que identificaran un aspecto relacionado con la salud que consideraran necesario abordar en su sección. La selección del tema fue completamente libre y les permitió identificar alguna problemática de salud existente dentro de su grupo de compañeros.

Durante esta primera semana, la docente presentó conceptos básicos de teorías del comportamiento aplicadas a la salud y, entró en detalle en el cómo y por qué desarrollar acciones de prevención de la enfermedad y promoción de la salud, mediante el uso de clases magistrales utilizando material audiovisual. Al final de esta semana, los estudiantes y las estudiantes crearon un primer avance de los objetivos y metodología a seguir en su proyecto.

Durante la segunda semana, el estudiantado trabajó de forma independiente, con el apoyo de la docente, en la mejora de objetivos y metodología, así como en la planificación de las actividades a realizar en una tercera y última sesión. Esto incluyó el desarrollo de materiales y la logística de las acciones a realizar. El estudiantado presentó, de forma verbal, sus avances a la docente del curso. Es importante destacar que se mantuvo siempre abierto un espacio de consulta entre estudiantes y docente durante el transcurso de la segunda y tercera semanas; esto, con el fin de facilitar la solución de problemas que se pudieran presentar con el desarrollo de las actividades propuestas.

Finalmente, en la tercera semana, se llevó a cabo la implementación de las intervenciones por parte del estudiantado. Cada grupo contó con 15 minutos para presentar de forma activa a sus compañeros y compañeras la problemática identificada y realizar una breve intervención de salud pública. Se esperó que los grupos estudiantiles fuesen claros en la presentación de sus objetivos y en la evaluación de las actividades. Como conclusión, se procedió a realizar una discusión sobre la importancia de identificar aspectos relacionados con la salud en los sitios de trabajo o en las comunidades donde se labora, como parte indispensable del accionar profesional en farmacia. 
Para la evaluación de las percepciones del estudiantado, se elaboró un cuestionario con preguntas abiertas y cerradas que fue enviado vía correo electrónico a cada estudiante. Este formulario se desarrolló utilizando el software en línea Survey Monkey ${ }^{\circledR}$, manteniendo aspectos de diseño del instrumento que garantizaran el anonimato de las personas que brindaron su respuesta. Los datos se analizaron utilizando Microsoft Excel ${ }^{\circ} 2007$ y son presentados en términos de frecuencias dada la baja cantidad de participantes. Así mismo, se realizó una descripción cualitativa de algunas de las respuestas recibidas.

\section{Resultados}

\section{Papel del personal docente}

La docente del curso asumió un rol de guía en la presentación de conceptos teóricos, en el desarrollo de los proyectos y en la presentación y evaluación de estos. Durante la etapa de planeación, la docente presentó aspectos básicos de promoción de la salud y prevención de la enfermedad, además explicó la metodología y objetivo buscado con el uso de la estrategia didáctica.

Por otro lado, al utilizar la estrategia de AOP, se proporcionó apoyo en las etapas de diseño y evaluación del proyecto. Esto incluye colaboración en el acceso a información, guía para estudiantes en la aclaración de sus dudas, retroalimentación, valoración del progreso y evaluación de los resultados de forma general.

\section{Papel del estudiantado}

Los estudiantes y las estudiantes se consideraron desde el principio del proyecto como sujetos creadores e innovadores. Fueron, además, ejes fundamentales en la identificación de una situación específica en su grupo, la cual podría ser considerada para la elaboración de su proyecto.

Se fomentó que el estudiantado mantuviera un pensamiento crítico en todo el proceso. Lo anterior con el fin de conservar la motivación durante el desarrollo de la actividad grupal. El estudiantado creó material e identificó los medios necesarios para la puesta en marcha de las actividades que su grupo decidió realizar para abordar un tema específico, y con el cual pudieran ejemplificar propuestas para promover estilos de vida saludables.

$\mathrm{El}$ accionar del estudiantado estuvo presente en casi todas las etapas del desarrollo del proyecto. Desde la planeación, hasta la construcción y puesta en práctica de las actividades estuvo completamente a su cargo. Además, tuvo un papel activo en la evaluación al participar en la retroalimentación de las actividades realizadas por sus compañeros y compañeras, y al responder encuestas proporcionadas por la docente en relación con el uso de la metodología. 


\section{Papel del contexto}

Dentro de los aspectos a considerar como parte del contexto físico, es importante mencionar que el aula donde se desarrolló la clase fue el aula 212 de la Facultad de Farmacia. Esta es el aula más grande del edificio y dado que la población matriculada en el curso fue de 28 , este espacio fue suficiente para el desarrollo de diversas actividades. La ventilación y la iluminación fue aceptable.

Como se indica en la introducción de este documento, el estudiantado que participó de este proyecto se encontraba en un periodo de sus estudios muy demandante. Debía transportarse de los sitios de práctica clínica (por ejemplo: hospitales nacionales) a el campus universitario en periodos cortos de tiempo y, en muchos casos, su llegada se daba minutos después de iniciada la clase. Además, fue posible identificar la presencia de problemas propios de la dinámica de grupo con diferencias marcadas entre estudiantes.

\section{Resultados y discusión}

En general, el estudiantado presentó una percepción positiva frente a la realización de esta actividad. Dentro de las bondades reportadas en mayor grado por estudiantes se encuentra que la elaboración de este proyecto les permitió poner en práctica acciones relacionadas con temas como la promoción de la salud y la prevención de la enfermedad. Lo anterior siguiere que la metodología utilizada favorece el cumplimiento del objetivo propuesto.

Al finalizar las tres semanas del proyecto, algunas de las frases que apoyan la realización del mismo indican:

"[Estas actividades] Ayudan a ejemplificar la teoría"

"Se pone en práctica los conceptos"

"Se aprende haciendo"

“...ponen a pensar como desenvolverse en una situación específica real”

Estas respuestas, además de mostrar una percepción positiva por parte del estudiantado, son apoyo para evidenciar la necesidad de marcar el vínculo existente entre aspectos teóricos y prácticos. Más aún, el apoyo mostrado por el estudiantado a esta actividad constituye un reto para que el personal docente promueva espacios que permitan la resolución de problemas, especialmente si están relacionados con su futuro ejercicio profesional.

Dentro de las limitantes para el desarrollo de la actividad se encuentra: el poco tiempo para el desarrollo de actividades y la baja asistencia con la que se contaba en algunos periodos del semestre. El tiempo para realizar las actividades está relacionado con el hecho de que el curso fue compartido por diferentes docentes lo cual limitó considerablemente la cantidad de sesiones disponibles para el desarrollo del proyecto por parte de la docente. Al respecto, se 
debe destacar que el contar con mayor espacio en el programa del curso podría permitir un mayor acompañamiento del proceso de aprendizaje del estudiantado y brindaría, además, mayor espacio para el trabajo del proyecto durante las horas lectivas, dada la reconocida complejidad de su carga académica del último semestre de la carrera de Farmacia.

Por otro lado, factores como la dificultad para transportarse desde los hospitales, donde recibían sus clases previas hasta la Universidad, provocaba que además de llegar una vez iniciada la sesión, estuvieran cansados y no se encontraran interesados en participar de las lecciones. Finalmente, al no ser el curso de asistencia obligatoria, y dado que la actividad a realizar no contaba con un porcentaje de la evaluación, podría ser causante de desmotivación. El siguiente comentario donde un estudiante se refiere, durante la primera semana, a la expectativa que tiene de la actividad que se les ha propuesto, evidencia lo mencionado anteriormente:

"Poca [expectativa], veo a la gente con pocas ganas de hacerla debido a que no hay calificación de por medio, creo que aunque suene feo, siempre es necesaria una evaluación al estudiante para que se esmere más al hacer un trabajo, y la distribución de puntos para la calificación en este caso me parece muy mala."

Sobre la distribución de la calificación, el comentario anterior hace referencia al curso en general y no al proyecto descrito en este documento. Sin embargo, permite reconocer la importancia que tiene para el estudiantado la evaluación de las diferentes acciones que se desarrollan, y que para sus intereses, su desempeño debe aparecer reflejado en la nota final del curso.

Interesantemente, y coincidiendo con lo observado en estudios que han profundizado en los altos niveles de estrés que poseen la población estudiantil de la carrera de Licenciatura en Farmacia de la Universidad de Costa Rica (Jiménez, 2010), la mayoría de los grupos coincidió, en la selección de temas para el desarrollo de sus proyectos, aquellos que tuvieran relación con el manejo del estrés y la mejora de las relaciones interpersonales. Las intervenciones breves realizadas para finalizar el proyecto incluyeron técnicas de relajación, organización del tiempo y manejo de las relaciones interpersonales.

\section{Valoración de la estrategia desde la perspectiva estudiantil}

Los estudiantes y las estudiantes respondieron, de forma voluntaria, un cuestionario antes y después de realizados los proyectos. En la consulta antes de la realización de sus proyectos (semana 1) sobre cuál era su expectativa de las actividades a realizar $(n=16)$, se destaca la utilidad de los proyectos $(\mathrm{n}=4)$, así como el dinamismo de de estos dentro de los puntos más importantes para el estudiantado $(n=9)$. Lo anterior permite considerar que, en general, la promoción de una mayor participación para el desarrollo de los contenidos es bien recibida por el estudiantado, destacando que por medio de acciones pueden mejorar el aprendizaje de conceptos teóricos. Dentro de las respuestas que reflejaban tener poca expectativa de las acciones a desarrollar $(\mathrm{n}=2)$ se incluyó la no consideración de la misma dentro de la evaluación del curso y en otro caso solamente se indicó que no consideraba que tuviera un impacto positivo en el aprendizaje. 
Trillo, Porto, Stewart y George (2014), al consultar a estudiantes sobre diversos aspectos relacionados con el proceso de evaluación, resaltan cómo se asocia la evaluación principalmente con la aplicación de pruebas y la obtención de un resultado. Esto es importante de considerar, ya que deberá ser una función docente el destacar el valor que tiene el desarrollo de este tipo de metodologías dentro del proceso de enseñanza aprendizaje, aunque no medie una prueba o un examen para su verificación, sabiendo que esto necesitará un cambio de paradigma por parte del estudiantado que está acostumbrado al sistema de evaluación tradicional de la Universidad.

Aunado a lo anterior, a la consulta sobre la utilidad de estos proyectos antes de su realización, 12 de 16 estudiantes expresaron que esperaban que este proyecto les ayudara mucho o algo en el aprendizaje de los conceptos de prevención de la enfermedad y promoción de la salud. Por otro lado, solamente tres participantes de esta encuesta consideraron que el uso de esta estrategia sería innecesario en su proceso de aprendizaje.

Con el afán de confirmar las percepciones de utilidad después de realizada la actividad, el estudiantado respondió la misma pregunta posterior a la realización de los proyectos. En esta ocasión, se obtuvo 12 respuestas y de ellas 10 indican que la realización de estos proyectos les ayudó mucho o algo en el proceso de aprendizaje y solamente 1 de ellos lo consideró innecesario.

La percepción de utilidad se mantuvo después de realizados los proyectos, lo cual refleja que, en este caso, el estudiantado ve reforzados los conceptos teóricos con la presencia de estrategias didácticas que permitan aprender por medio de la práctica. Esto es apoyado por la opinión estudiantil, al afirmar que este tipo de actividades permite ejemplificar conceptos teóricos que son importantes para su aprendizaje y su formación profesional.

Finalmente, se le preguntó: ¿qué le gustó de la sesión de presentación de los proyectos? De las respuestas brindadas se presenta una que resume la mayoría de los aportes:

"El hacer de manera muy sencilla una pequeña intervención la cual nos acerca a lo que nos enfrentaremos en un futuro, asi como los lineamientos para hacerla, ya que uno a veces piensa que lo está haciendo bien pero se hace muy desordenado"

Esta frase refleja que la intención de la utilización del AOP para el desarrollo de los contenidos de promoción de la salud y prevención de la enfermedad en el curso de Salud Pública de la Facultad de Farmacia fue exitosa. Permite, además, ejemplificar la necesidad de reforzar contenidos teóricos con la práctica y la identificación de espacios para el desarrollo de intervenciones dentro de las comunidades.

Considerando lineamientos de la Organización Mundial de la Salud (2013), podemos indicar que los resultados sugieren la importancia de fomentar en el estudiantado el desarrollo de acciones que refuercen el aprendizaje de contenidos teóricos; pero que, además, les inviten a ser parte de sus comunidades y conocer las necesidades, para que así quien se dedique a la profesión de farmacia tenga mayor conciencia social y se involucre en ellas para la mejora de la salud de todas las personas (OMS, 2013). 


\section{Conclusión}

La estrategia de aprendizaje orientado en proyectos fue bien recibida por los estudiantes y las estudiantes del curso. El uso de estrategias didácticas que le vinculen con la realidad y le reten a ser miembro activo dentro de su comunidad para colaborar en la resolución de necesidades específicas es un elemento a considerar como parte de su formación como profesionales del futuro en salud. Igualmente, la identificación de problemáticas de salud le permitirá poder planificar adecuadamente las acciones a desarrollar y le guiará en su compromiso por la mejora de la salud.

El uso del aprendizaje orientado en proyectos permitió presentar contenidos de una manera aplicada y considerando aspectos de la realidad en la cual se desenvuelven el estudiantado. Es necesario que este tipo de actividades se continúe realizando teniendo consideraciones de tiempo para que así se pueda disponer de un mayor espacio para la planificación del proyecto. Se puede también considerar otros espacios o comunidades para el desarrollo de las acciones por parte de estudiantes de niveles avanzados de la carrera de Licenciatura en Farmacia.

Con el fin de aumentar el compromiso estudiantil, es necesario considerar este proyecto como parte integral de la evaluación a realizar en el curso, ya que este fue uno de los factores más importantes mencionados por el estudiantado cuando se le solicitó retroalimentación de manera verbal y escrita.

\section{Referencias}

Baños, J. y Pérez, J. (2005). Cómo fomentar las competencias transversales en los estudios de ciencias de la salud: Una propuesta de actividades. Educación Médica, 8(4), 216-225.

Blumenfeld, P. C., Soloway, E., Marx, R. W, Krajcik, J. S., Guzdial, M. y Palincsar, A. (1991). Motivating Project-Based Learning: Sustaining the doing, suppporting the learning [Motivando el aprendizaje basado en proyectos: Nutriendo el hacer, apoyando el aprendizaje]. Educational Psychologist, 26 (3 y 4), 369-398. doi: http://dx.doi.org/10.10 $\underline{80 / 00461520.1991 .9653139}$

Currás, M. (1999). Percepción de los estudiantes sobre su evaluación en la Universidad. Un Estudio en la Facultad de Ciencias de la Educación. Innovación Educativa, 9, 55-75.

Divine, H. S., y Cain, J. (2009). Assessing the Effect of a Polypharmacy Medication Adherence Simulation Project in a Geriatrics Course in a College of Pharmacy [Valoración del efecto de un proyecto de simulación para la adherencia a medicamentos en pacientes polimedicados en un curso de geriatría en una escuela de Farmacia]. J Am Geriatr Soc, 57 (8), 1487-1491. doi: http://dx.doi.org/10.1111/j.1532-5415.2009.02364.x 
Dobson, R., Taylor, J., Henry, C., Keegan, D. y Forbes, D. (2009). Taking the lead: Community pharmacists'perception of their role potentian within the primary care team [Tomando el liderazgo: Percepciones de farmacéuticos de comunidad sobre su rol potencial dentro del equipo de atención primaria]. Research in Social and Administrative Pharmacy, 5, 327-336. doi: http://dx.doi.org/10.1016/j.sapharm.2008.11.002

Escribano, A. y Del Valle, Á. (2008). El aprendizaje basado en problemas. Una propuesta metodológica en educación superior. Madrid, España: Narcea.

Fuhrman, L. C., Buff, W. E., Eaddy, M. y Dollar, M. (2001). Utilization of an Integrated Interactive Virtual Patient Database in a Web-Based Environment for Teaching Continuity of Care [Uso de una base de datos virtual interactiva de pacientes en un ambiente en internet para la enseñanza del cuidado continuo] American Journal of Pharmaceutical Education, 65(3), 271-275. Recuperado de http://archive.ajpe.org/legacy/ pdfs/aj650313.pdf

Galeana, L. (s. f.). Aprendizaje basado en proyectos. Recuperado de http://ceupromed.ucol.mx/ revista/PdfArt/1/27.pdf

Jiménez Herrera, L. G. (2010). Estrés en estudiantes de farmacia de la Universidad de Costa Rica. Actualidades Investigativas en Educación, 10(2), 1-29.

Organización Mundial de la Salud. (2013). Servicios farmacéuticos basados en la atención primaria de salud. Serie La renovación de la atención primaria de salud en las Américas. Número 6. Washington, D.C.: Autor.

Rodríguez-Sandoval, E., Vargas-Solano, É. M., y Luna-Cortés, J. (2010). Evaluación de la estrategia "aprendizaje basado en proyectos". Educación y educadores, 13(1), 13-25. doi: http://dx.doi.org/10.5294/edu.2010.13.1.1

Tan, E., Stewart, K., Elliott, R. y George, J. (2014). Pharmacists services provided in general practice clinics: A systematic review and meta-analysis. [Servicos farmacéuticos brindados en clìnicas de práctica general: Una revisión y meta-análisis]. Research in Social and Administrative Pharmacy, 10(4), 608-622. doi: http://dx.doi.org/10.1016/j. sapharm.2013.08.006

Trillo Alonso, F., Porto Tan, E., Stewart, K., Elliott, R. y George, J. (2014). Pharmacists services provided in general practice clinics: A systematic review and meta-analysis. [Servicos farmacéuticos brindados en clìnicas de práctica general: Una revisión y metaanálisis]. Research in Social and Administrative Pharmacy, 10(4), 608-622. doi: http:/l dx.doi.org/10.1016/j.sapharm.2013.08.006 